\title{
Introducing Online Quizzes into Lab-Based Teaching in University
}

\author{
Ying Cheng, Shaofeng Lu, Yang Du, and Gee Lim
}

\begin{abstract}
ICE is a virtual learning environment developed based on Moodle and currently heavily applied at Xi'an Jiaotong-Liverpool University (XJTLU). ICE is found to be an effective tool to enhance the teaching and learning experiences for both teachers and students. In this paper, we propose to introduce online quizzes on ICE into the lab-based teaching to address three existing issues in current lab-based teaching practices. These issues include a lack of initiative for lab preparation for students, missing out key information during the lab-teaching process, and insufficient support for lab instructors on planning the lab contents based on students' understanding on key theoretical concepts. We expect that the online quizzes based on ICE will motivate students, reinforce their knowledge basis to conduct the lab experiments and facilitate lab instructors to make a better lab teaching plan according to students' understanding on the lab contents. Further evidences are yet to be found to understand the benefits of the designed online quizzes.
\end{abstract}

Index Terms-Moodle, ICE, online quizzes, lab-based teaching.

\section{INTRODUCTION}

Modular Object-Oriented Dynamic Learning Environment (Moodle) is a virtual learning environment designed to provide educators and learners with a robust and integrated system to create personalized learning experiences. Its official language is English and it is open source under the General Public Licence (GPL). There is a potential to extend or modify Moodle for teaching process or other projects. Because of the low cost, flexibility and other advantages, a large number of institutions and schools around the world are in favour of Moodle and have adopted it to satisfy their own teaching and learning demands [1]-[3]. It has been the most widely used learning platform with 79 million users [4]. Moodle was first introduced into China in 2004 and many papers proposing to integrate Moodle with teaching activities have been published ever since [5], [6]. East China Normal University introduces an assessment based on Moodle [7]. Shanxi Normal University analyses the factors that influence the interaction between teachers and students on Moodle [8]. There are also several schools and colleges which have applied Moodle to some curriculums in Chinese context [9]-[12]. Xi'an Jiaotong-Liverpool University (XJTLU) is amongst the earliest universities in China adopting Moodle (ICE) to enhance its teaching and learning systems.

Manuscript received April 7, 2016; revised August 16, 2016.

The authors are with the Department of Electrical and Electronic Engineering, Xi'an Jiaotong-Liverpool University, China (e-mail: Cheng.Ying@xjtlu.edu.cn, Yang.Du@xjtlu.edu.cn, enggee.lim@xjtlu.edu.cn).
Located in Suzhou, Jiangsu Province, XJTLU is an international university founded by Xi' an Jiaotong University in China and the University of Liverpool in the UK as a joint venture in 2006. To realize its ambition, which is "to provide a unique international learning environment, drawing on the strengths of Chinese and Western traditions and best practices in global higher education", the university encourages tremendous educational explorations and experiments since the first day when it was founded. ICE, as a learning management platform developed based on Moodle and specially tailored for learning and teaching needs on campus, is one of the many explorations to support and enhance learning and teaching at XJTLU. In this paper, we would like to study how teaching activities could benefit from the support of ICE. To be specific, how on-line quizzes, one of the many powerful functions on ICE may solve some existing issues arising from our current engineering lab-based teaching practices.

\section{ISSUES ON CURRENT ENGINEERING LAB-BASED TEACHING}

Lab-based teaching in which hands-on experiments are to be conducted by students takes an important part for a wide range of disciplines. In current practice, lab-based teaching usually involves live demonstrations and hands-on experiments. But there are three major issues in the lab-based teaching at XJTLU.

Firstly, there is a lack of effective way to improve the initiative of students for preparation of the lab. XJTLU has a free style campus environment and hopes that the lab preparation could be managed based on students' self-discipline. However, students tend to spend less time on lab preparation and wait for the information to be fed in the process of live demonstrations which causes low learning efficiency during the lab.

Secondly, key information is easy to be missed or misunderstood via traditional learning channels, such as reading lab manuals before the lab. Since all the programs are taught in English at XJTLU, including lecturing languages and learning resources, it would become a less exciting and sometimes less efficient task to understand the lab manuals to prepare the lab. When the lab manual contains too much detailed information to get them well instructed, students probably miss out some key information along the whole lab procedure.

Thirdly, insufficient information is collected to evaluate students' understanding on the important concepts which may be heavily involved during the lab. It is hard to know how well the students have prepared the lab without a proper 
evaluation method in our current practices. Therefore, it is difficult for the lab instructor to provide more responsive lab instructions during the live demonstrations and this would probably lead to a lower teaching and learning efficiency during the lab.

Actually, some researches have presented a general idea of integrating Moodle with lab-based teaching [2], [13], [14]. They demonstrate the meaning and potential effects of the integration of Moodle, but none has applied such integrations in practice yet. As mentioned above, XJTLU already has relative mature practices of adopting Moodle to curriculums. It's practical to realize the idea based on our actual experiences and requirements. So we propose to introduce well-designed online quizzes based on ICE into our current engineering lab-based teaching to improve and enhance the learning experiences for students with better support. These online quizzes can be easily incorporated into our current assessment system and thus provide a stimulation on students' initiative for the preparation of the lab. Key information can be effectively conveyed using the highly tailored feedbacks provided by the online quizzes. Using the assessment statistics collected after the quizzes, lab instructors would generate better responsive lab teaching plan to support the learning process of students.

\section{A SYSTEMATIC DESIGN ON ONLINE QUIZZES IN LAB-BASED TEACHING}

Teaching and learning efficacies in labs depend directly on how well students are prepared before the experiments and how much they critically think and summarize after lab experiments. In general cases, students may be asked to submit pre-lab reports to show they have read lab manuals and final reports containing analyzed statistics after experiments. In this way, we assume that students have mastered the knowledge well. As mentioned above, there are still some unsolved problems that we hope to solve by introducing ICE and online quizzes. This process of integration could be divided into three phases as shown in Fig. 1.

Phase I: ICE is the platform where students get detailed instructions for experiments and take quizzes before field experiments. Teachers upload all the teaching resources, including lab regulations, experimental principles and background, operational procedures and video demonstration. Then a carefully designed and time-limited quiz will be released. After that teachers could learn how well students master the essential knowledge for conducting experiments by the results of the quiz. Students need to take some time to learn relevant resources and then take the online quiz. They could access ICE anytime, anywhere through ICE app or website before experiments. Students could learn and prepare themselves before the field experiments and they are welcome to try several times to retake the quiz. But only those who pass the online quiz are allowed to attend hands-on experiments in lab arranged later than the quizzes. Alternatively, penalties can be applied for those who have not completed the online quizzes.

Phase II: Field experiments will be conducted by students in the laboratory under supervision. Students could still get immediate live guidance if they come across any questions. They are expected to take the initiative and finish experiments independently in the laboratory in the first place.

Phase III: After the experiments, students could discuss questions in the forum on ICE. Then they must submit a final report or take a final test. Teachers could obtain statistic of grades showing how well students master hands-on skills and how much they learn about experiments based on the report or the final test.

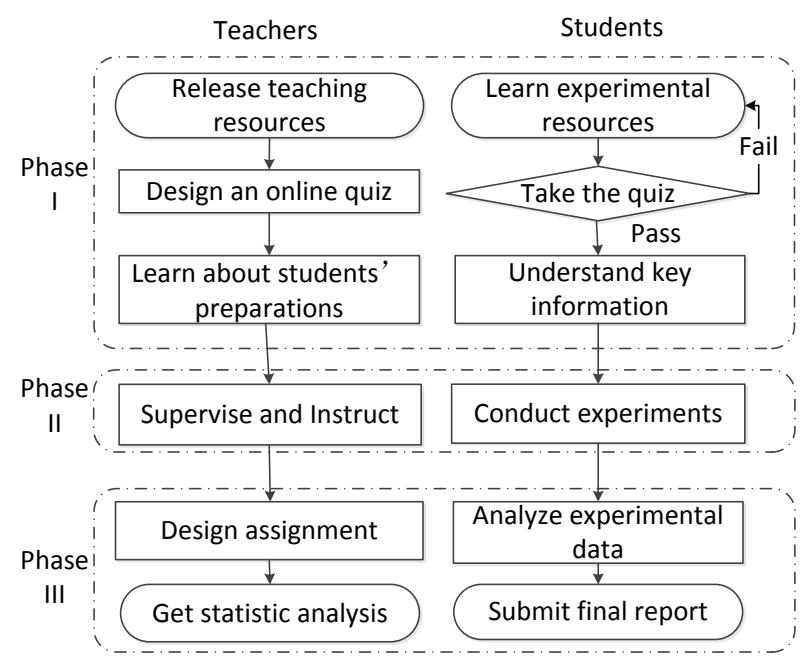

Fig. 1. Three phases of lab-based teaching when cooperating with ICE and online quizzes.

\section{QUIZ QUESTIONS AND STATISTIC ANALYSIS ON QUIZ RESULTS}

The most important advantage of online quizzes introduced into lab-based teaching is that it could provide immediate and personalized feedbacks. Feedbacks could motivate students and influence their understanding of knowledge. Feedbacks will also make students aware of what they are doing and give directions for what to do next. For teachers, on the other hand, they can get a macroscopic view of students' quiz performances and make a better teaching plan.

There are a variety of question types of online quizzes that could be used on ICE, such as numerical questions, calculated questions, embedded answers, drag and drop matching, ordering, true or false, multiple choices, essays and so on. Different types could be chosen to make up a quiz according to actual requirements. The numerical question allows a numerical response, possibly with a unit, which is graded by comparing against various model answers. The calculated question is similar to the numerical question but with variables used selected randomly from a set when the quiz is taken, which could avoid plagiarism to some extent. Multiple choices allow the selection of a single or multiple responses from a pre-defined list. For these question types, Allows a response of a few sentences or paragraphs. This must then be graded manually Allows a response of a few sentences or paragraphs. This must then be graded manually Allows a response of a few sentences or paragraphs. This must then be graded manually the platform could mark students' answers automatically and thus improve teaching efficiency. The essay, on the other hand, allows a response of a few sentences 
or paragraphs, which must then be graded manually and provide a possibility of implementing the traditional assessment.

As stated above, we hope to give personalized feedbacks to students when they are taking quizzes. We could realise the objective by setting up multi-level feedbacks to each question. For the calculated question whose variables are selected randomly from a set, we could set up separate feedbacks for each answer. More specifically, a general feedback will be shown right away if the answer is wrong. It may contain relative equations, or explain a theory or a clue to the right answer. A specific feedback will show the process of solving the problems if the answer is correct. Some other feedbacks could also be added if similar mistakes are frequently made on a confusing question, which will help students figure out how they get confused on certain steps.

If the question is multiple-choice type, we could set up feedbacks to each choice. Below, we will demonstrate a case study to explain the feedback system using a multi-choice question example. The question is based on a year-one module on electrical circuit analysis in the Department of Electrical and Electronic Engineering at XJTLU. The lab is about demonstrating the effect of Thévenin's Theorem. When conducting the lab, students usually find it challenging as the contents are only covered briefly during the lectures beforehand. So we design the questions with different feedbacks available to prepare students on the theoretical background on Thévenin's Theorem. We will use screen snapshots of the online quizzes to illustrate the functions of a multi-choice question.

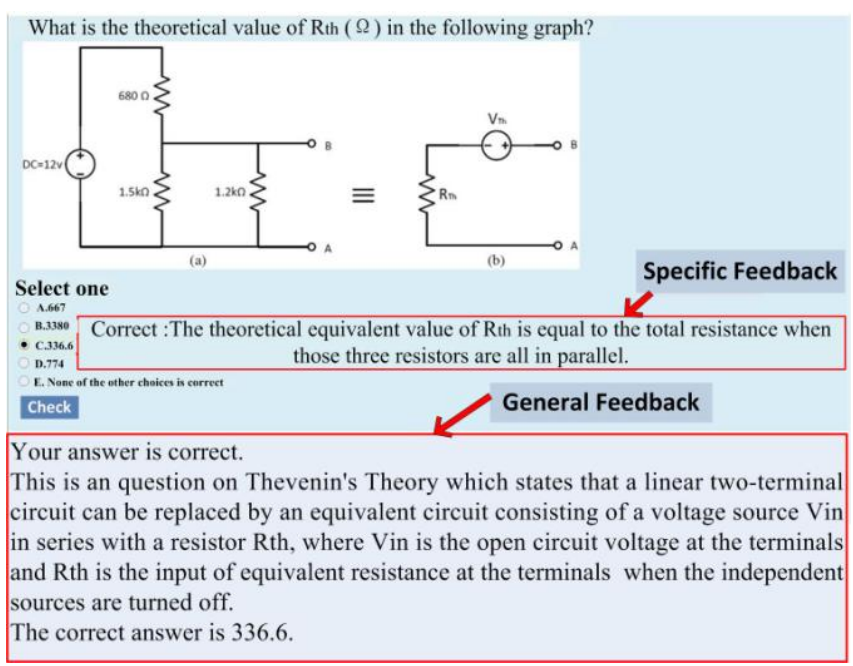

Fig. 2. Feedbacks on each question choice.

As shown in Fig. 2, there are two types of feedbacks, i.e., specific feedback and general feedback, on the question asking about Thévenin equivalent resistance. Note that this figure is the same setup circuit used for the lab. By doing so, we try to facilitate students on better preparations on the lab experiments. The specific feedback will take care of each choice and try to respond to any potential reasons why students take this choice, whether it is correct or incorrect. One of the incorrect cases is demonstrated in Fig. 3.The general feedback put the question background and core assessment contents in detail. In this question, we have included the Thevenin's Theorem for students' information so that students can quickly review the fundamental theorem during the process of solving this question.

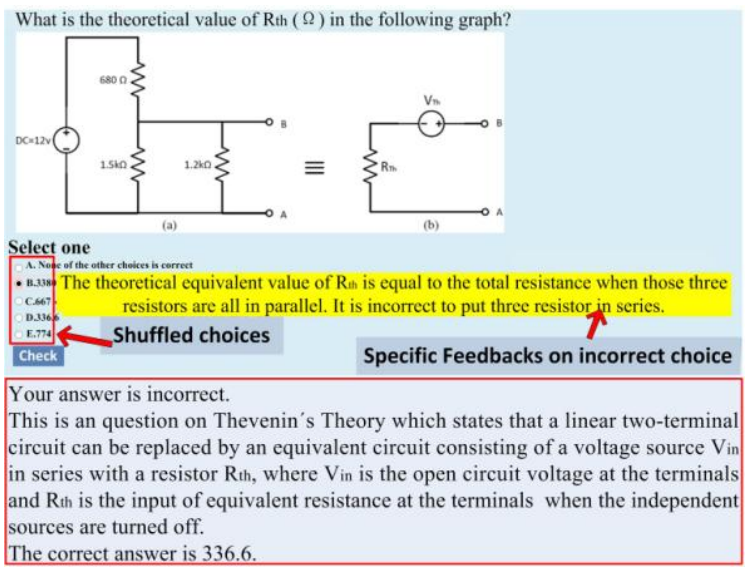

The correct answer is 336.6

Fig. 3. Shuffled choices in the question and specific feedback for incorrect answer.

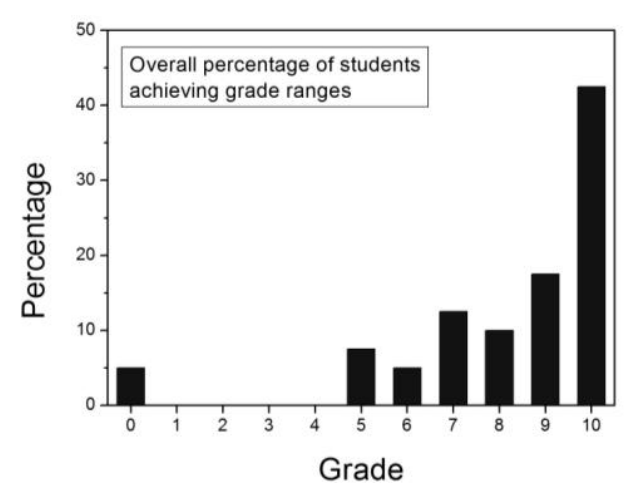

Fig. 4. Overall percentage of students achieving various grades.

In Fig. 3, it is seen that the specific feedback on choice B has been designed to reflect the possible causes behind this choice. It is speculated that any student who chooses choice B has chosen the answer by putting all three resistors into series which is against the Thévenin Theorem. The specific feedback explicitly points out the mistake so that it makes sure students understand what goes wrong.

It is worth mentioning that one of the powerful functions for online quizzes based on ICE is that the choices will be shuffled from one attempt to another. As shown in Fig. 3, it is noticed that the choices available have been shuffled from the ones in Fig. 2. This generally prevents students from manipulating the answers. Ideally, even two students sitting next to each other and taking the same quiz question, the answer can be different by careful design. In summary, combining both specific feedbacks and general feedbacks based on different type of questions, each quiz question becomes an interactive learning resource to prepare students in the lab session.

Teachers could also get statistics from the grade report of the quizzes. These results contain the number of students' quiz attempts, the overall grade for each student, and the grade for each question. As an example from a previous online quiz, the overall percentage of students achieving grade ranges is displayed in a bar chart in Fig. 4. We can easily spot that more than $40 \%$ of students have achieved a full grade which is equivalent to 10 and nearly $5 \%$ of total students only achieve a grade of 0 indicating no response or an ineffective response to the quizzes. The overall average of each question is also 
shown in Fig. 5 and it is found that Question 7 is found to be most challenging in comparison with other questions.

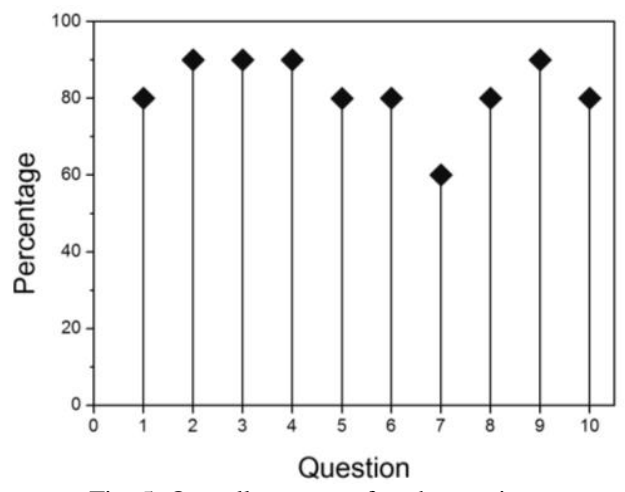

Fig. 5. Overall average of each question.

The statistic is useful for teachers because it helps them make a better responsive teaching plan and therefore they could explain relative knowledge to students in class afterwards. Teachers could also check on an individual student's behaviour on each question and how much time the student has spent on it.

\section{CONCLUSIONS}

In this paper, we proposed to introduce online quizzes on ICE, into lab-based teaching to address three typical existing issues. Not only would ICE improve teaching efficiency by automatically mark students' answers and provide responsive multi-level feedbacks, but also it could improve the intellectual interactions between teachers and students. Through a careful design on these online quizzes, we expect to strengthen the motivation of students on lab preparation and provide personalized comments and feedbacks to students. Students could learn about what are the essential concepts before experiments and also clarify doubts and confusions during the learning process. The statistics analysis on the quiz results will also support teachers to learn about students' performance on quizzes and make a better teaching plan accordingly.

In summary, equipped with well-designed online quizzes, improvements on the quality of the lab-based teaching and enhancement on the students' learning experiences are to be expected. The future work of this project is to collect more solid evidence to understand how much benefits these online quizzes could add into our current lab-based teaching.

\section{ACKNOWLEDGMENT}

The project is supported by Teaching Development Fund (TDF) at XJTLU (Project No. 14/15-R10-087).

\section{REFERENCES}

[1] M. A. Trenas, J. Ramos, E. D. Gutiérrez, S. Romero, and F. Corbera "Use of a new moodle module for improving the teaching of a basic course on computer architecture," IEEE Transactions on Education, vol. 54, pp. 222-228, May 2011.

[2] K. Seluakumaran, F. F. Jusof, R. Ismail, and R. Husain. "Integrating an open-source course management system (Moodle) into the teaching of a first-year medical physiology course: A case study," Advances in Physiology Education, vol. 35, pp. 369-377, Dec. 2011

[3] P. A. Rodrigues, L. O. Brandão, and A. A. F. Brandão. "Interactive assignment: A moodle component to enrich the learning process," presented at $40^{\text {th }}$ ASEE/ IEEE Frontiers in Education Conference, Washington, DC, October 27-30, 2010

[4] M. Dougiamas. About Moodle. [Online]. Available: https://moodle.org/

[5] S. Luo and J. H. Li, "Linking classroom and outside study with moodle," Front Line of the Theory, vol. 2, pp. 4-7, Apr. 2007.

[6] S. Q. Liu and J. H. Li. "Curriculum design BIG6 mode based on moodle," Distance Education Journal, vol. 1, pp. 4-8, Feb. 2007.

[7] X. L. Chen, Z. Y. Chen, and X. H. Wang. "Teaching assessment based on moodle," Computer Education, vol. 8, pp. 68-71, Apr. 2007.

[8] J. Qin, X. H. Du, and H. L. Ma. "Empirical analysis on the interactivity of learning management platform of moodle," China Educational Technology, vol. 2, pp. 86-90, Feb. 2011.

[9] L. Peng, Y. H. Mo, and W.Q. Xian, "Classroom response system based on moodle," Distance education Journal, vol. 3, pp. 68-70, June 2008.

[10] X. Y. Zhang and Z. Q. Ma, "Review of the research on platform of moodle," Open Education Research, vol. 13, pp. 96-99, Dec. 2007.

[11] J. P. Ouyang and Q. J. Li, "Construction of school-based curriculums using LMS system," CET China Educational Technology, vol. 4, pp. 61-64, Apr. 2007.

[12] R. L. Wang, M. J. Li, and Y. H. Ma, "Web-based cooperative learning on physics classes in middle school based on moodle," CET China Educational Technology, vol. 8, pp. 57-60, Aug. 2006.

[13] S. P. Pan and Q. Wang, "The application of moodle in experimental teaching in colleges and universities," Experimental Technology and Management, vol. 26, pp. 90-93, Oct. 2009.

[14] Y. K. Lai and B. Y. Wu, "Innovation explorations on lab-based teaching in universities based on moodle," Computer Knowledge and Technology, vol. 8, pp. 2802-2804, Apr. 2012.

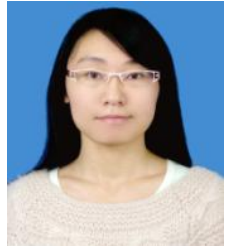

Ying Cheng obtained the degrees of BEng in electrical engineering and MSc in optical engineering from Xiamen University in 2011 and 2014 respectively. She is a lab technician with the Department of Electrical and Electronic Engineering at XJTLU in Suzhou. Her research interests include: Q-switched microchip lasers, education technologies for lab-based teaching.

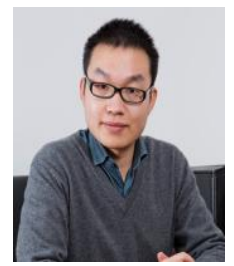

Shaofeng Lu received the degrees of BEng and $\mathrm{PhD}$ from the University of Birmingham UK, in 2007 and 2011 respectively. He also has a BEng degree from Huazhong University of Science and Technology (HUST), China. All are in electrical and electronic engineering.

Dr. Lu is a lecturer with the Department of Electrical and Electronic Engineering at XJTLU in SUZHOU. Dr. Lu is devoted to intelligent energy efficiency improvement technology of railway traction systems and smart grid energy systems. He also has research interests in engineering education practices.

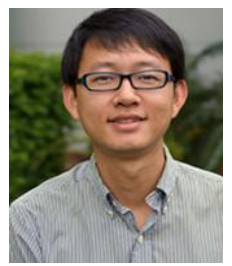

Yang Du received his $\mathrm{Ph} . \mathrm{D}$. degree in electrical engineering from the University of Sydney, Australia, in 2013 .

From 2013 to 2014, he was with Masdar Institute, Abu Dhabi, UAE, as a post-doctoral research fellow. Currently, he is a lecturer at Xi'an Jiaotong-Liverpool University, Suzhou, China. His research interests include power electronics application in photovoltaic system, power quality issues caused by PV inverter and reliability of power electronics converter.

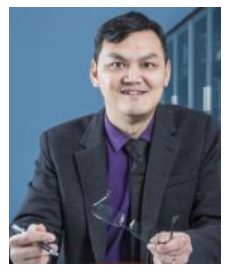

Gee Lim received the BEng(Hons) and $\mathrm{PhD}$ degrees in electrical and electronic engineering from the University of Northumbria, UK in 1998 and 2002 respectively.

Prof. Lim worked for Andrew Ltd, a leading communications systems company in the United Kingdom from 2002 to 2007. Since August 2007, Prof. Lim has been with the Department of Electrical and Electronics Engineering, Xian Jiaotong-Liverpool University, where he is currently a professor and head of department. He has published over 100 refereed international journals and conference papers. His research interests are energy harvesting, power/energy transfer, antennas, $\mathrm{RF} /$ microwave engineering, EM measurements/simulations, and wireless communication network. 